\title{
Network Coding Schemes for the Multiple-Access Relay Channel
}

\author{
Stefan Dierks, Onurcan İşcan, Christoph Hausl \\ Lehrstuhl für Nachrichtentechnik \\ Technische Universität München \\ Munich, Germany \\ \{stefan.dierks, onurcan.iscan, christoph.hausl\}@tum.de
}

\begin{abstract}
We examine and compare four different joint-network-channel-codes (JNCC) and three reference systems for the multiple-access relay channel (MARC) with block Rayleigh fading channels for symmetric and asymmetric setups. Two of the JNCC's were presented previously in the literature and the other two are novel approaches. The JNCC's outperform the reference systems and achieve a diversity gain for symmetric and asymmetric channels, where the performance differences are greater for increasing asymmetries. An optimization of the symbol allocation using statistical channel knowledge is used, which generates a small gain in the theoretical bounds and for some of the practical JNCC's for asymmetric setups.
\end{abstract}

\section{Categories and Subject Descriptors}

E.4 [Coding and Information Theory]: Error control codes-Joint-Network-Channel-Codes

\section{General Terms}

Theory

\section{Keywords}

Cooperative Communications, Multiple-Access Relay Channel, Network Coding, Half-Duplex Relay

\section{INTRODUCTION}

To improve the performance of users which suffer from fading, diversity techniques can be used. In this work we use a relay and network coding to gain cooperative diversity. We present coding schemes for the multiple-access-relay-channel (MARC) [13]: The MARC can represent the uplink from mobile stations to a base station, where relays are used to support communication.

In this work we use time-division multiple access (TDMA), which means that only one station is allowed to transmit at one time instant, therefore a half-duplex relay is sufficient,

Permission to make digital or hard copies of all or part of this work for personal or classroom use is granted without fee provided that copies are not made or distributed for profit or commercial advantage and that copies bear this notice and the full citation on the first page. To copy otherwise, to republish, to post on servers or to redistribute to lists, requires prior specific permission and/or a fee.

VALUETOOLS 2011, May 16-20, Paris, France

Copyright (C) 2011 ICST 978-1-936968-09-1

DOI 10.4108/icst.valuetools.2011.245801 however TDMA is suboptimal regarding the capacity [13]. Capacity bounds and coding strategies for the MARC with half-duplex and full-duplex relays are given in [15].

We concentrate on decode-and-forward strategies, which are useful when the relays are located close to the source [12]. We give an overview over related work for decode-and-forward schemes: In [3] an analysis of the diversity gain is given for a separate-network-channel-code (SNCC). Here channel coding is used to create erasure-based links. The messages from the mobile stations are combined using linear network coding [1] in the relay. Using network coding allows to gain diversity for higher rates. In the base station erroneous message can be retrieved from the relay transmission by reversing the network code, when all other messages are decoded correctly. A different approach is taken in [22], where the relay is assumed to be noisy and not the network-coded messages are forwarded, but the network-coded log likelihood ratios (LLR) of the received transmissions. The LLR are used in the base station for an iterative cross-layer decoding with the direct transmissions. This allows to exploit the transmission of the relay as additional redundancy that supports the channel code for the error protection. Since both network and channel-coding are used jointly, it is called a joint-network-channel-code (JNCC). In [10] and [9] a JNCC for the MARC is presented, where the information bits are multiplexed into a new sequence in the relay. The received transmission of this sequence can be used in the base station together with the extrinsic information of the two direct transmission decoder to create a priori information that is fed to the direct decoders for the next iteration. In [21] and [20] codes to gain cooperative diversity are presented for a setup without a relay, where the mobile stations support each other in transmitting their messages to the base station. The authors use an approach where the stations transmit an algebraic superposition of their own encoded message and of the other interleaved encoded messages. The decoder in the base station uses a window of a number of received codewords to retrieve a message, where the number of states of the decoder is the product of the number of states of the individual encoders. The approach of these references could be applied to a MARC, too. Another approach, which can be applied to a MARC, is given in [11], where the code bits are network-encoded in the relay and the extrinsic information of a direct decoder is used to retrieve the information of the other message from the relay transmission. In [5] the authors present strategies to produce low-density parity-check codes for the MARC working at high rates and achieving 
full-diversity. For the semi-orthogonal and non-orthogonal MARC, where more than one node is allowed to send at the same time, JNCC's are presented in [7] and [8]. A summary of recent developments of cross-layer network coding is given in [14].

In this work we present four JNCC's: The approach from [10], where the information bits are multiplexed in the relay; An adapted version of [11], where we network-encode the code bits; A novel approach with a simple non cross-layer relay, which network-encodes the information bits, but with joint-network-channel-decoding at the base station; And a second novel approach similar to the first approach, where we use a duo-binary encoder instead of multiplexing. Additionally we present SNCC, relay channels [16] and pointto-point channels as reference systems. The main contribution of this work is the comparison of the four approaches for symmetric and asymmetric MARC's and an approach to optimize the symbol allocation to improve the results.

The structure of this document is as follows: Before the explanation of the JNCC schemes in Section 3 and the reference systems in Section 4, we explain in Section 2 the system model. In Section 5 we show the performances for symmetric and asymmetric channels of the presented schemes for a not optimized symbol allocation. To improve the performance the symbol allocation can be optimized as explained in Section 6 and the results using optimized symbol allocations are shown in Section 7. Finally a conclusion is given.

\section{SYSTEM MODEL}

We consider a multiple-access-relay-channel (MARC) [13] with two source node and one relay as depicted in Figure 1. We restrict our system to time-division multiple-access (TDMA) and hence a symbol allocation is needed which assigns each source and relay a unique time slot to transmit its symbols. This approach is suboptimal compared to the general MARC model [13]. However it does not require a full duplex relay, but only a half duplex relay, which eases a realization in practice [10].

\subsection{System Setup}

We denote the mobile stations as MS1 and MS2, the relay as $\mathrm{R}$ and the base station as BS. The $i$-th MS transmits statistically independent data segmented into packets $\boldsymbol{u}_{i}$ of length $K_{i}$ including the CRC bits. Each packet is protected by a channel code against transmission errors. If not stated otherwise we use the UMTS turbo code [17] in the mobile stations. The length of a encoded sequence $\boldsymbol{c}_{i}$ is $M_{i}$, which is also equal to the length of the transmitted symbol sequence $\boldsymbol{x}_{i}$, since we use BPSK as modulation alphabet in the mobile stations and the relay.

The relay receives disturbed versions of the encoded messages $\boldsymbol{x}_{1}$ and $\boldsymbol{x}_{2}$ and obtains the estimates of the information bits $\hat{\boldsymbol{u}}_{\mathrm{R}, 1}$ and $\hat{\boldsymbol{u}}_{\mathrm{R}, 2}$ by demodulating and decoding. The relay can determine with the help of the CRC if the decoding was successful. In case that both messages were decoded successful, the relay encodes them using channel and network-coding to obtain its transmission $\boldsymbol{x}_{\mathrm{R}}$ of length $K_{\mathrm{R}}$. The strategy on relay decoding errors that we consider is for the relay to remain silent. We treat the whole system as one network-channel-code with system rate $R_{\mathrm{S}}=\left(K_{1}+K_{2}\right) /\left(M_{1}+M_{2}+M_{\mathrm{R}}\right)=\left(K_{1}+K_{2}\right) / M_{\text {total }}$.

\subsection{Channel Model}

Each channel is a block Rayleigh-fading channel, where we assume that the channel realizations are independent. Since our channel models are memory-less and each packet experiences block fading, the output after the matched filter at node $j$, receiving a packet $\boldsymbol{x}_{i}$ from node $i$, can be written as:

$$
\boldsymbol{y}_{i, j}=h_{i, j} \boldsymbol{x}_{i}+\boldsymbol{z}_{i, j},
$$

where $h_{i, j}$ is the channel coefficient and $\boldsymbol{z}_{i, j}$ is the noise vector. The noise values are independent and identically distributed (iid) complex Gaussian random variables (RV) with zero mean and variance $\sigma_{n}^{2}$ which is the power of the noise. The channel coefficient between node $i$ and $j$ is given as:

$$
h_{i, j}=a_{i, j} \sqrt{\left(\frac{d_{0}}{d_{i, j}}\right)^{n}},
$$

where $a_{i, j}$ is the fading coefficient and the rest resembles the attenuation of the amplitude due to the path loss, which is determined by the distance between the two nodes $d_{i, j}$ and the reference distance $d_{0}$, which we set to 1 to ease further notation. The fading coefficient is circularly symmetric and its expectation is set to $\mathrm{E}\left[|a|^{2}\right]=1$. The absolute value of $a_{i, j}$ is Rayleigh distributed. It is assumed that the power at the receiver is normalized, which allows the path loss to be included in the variance of the noise. The average SNR does not change over time and is given as

$$
\rho_{i, j}=\frac{P_{i}}{d_{i, j}^{n} \cdot N_{0}} .
$$

Now we can write the instantaneous SNR as

$$
\gamma_{i, j}=\left|a_{i, j}\right|^{2} \cdot \rho_{i, j},
$$

which is valid for one block.

We denote, as can be seen in Figure 1 the average SNR of a MS-BS channel with $\rho_{\mathrm{MS}, \mathrm{BS}}=\rho$. The other average SNR's are specified by an offset from $\rho$, which is due to the different distances between the notes. To describe a non symmetric setup with regard to the MS-BS-channels we use the factor $\delta$. When $\delta$ is set to zero the channel setup is symmetric. For positive values of $\delta$ the SNR on the MS1-BS channel is decreased and on the MS2-BS channel it is increased.

\section{JOINT-NETWORK-CHANNEL-CODES}

We present in this section four JNCC's, in which the transmission from the relay is used not only to improve the diversity order, but also to gain additional redundancy for error protection. JNCC's are cross-layer codes and exchange soft information iteratively during the decoding in the base station.

\subsection{JNCC I: Multiplexing Information Bits}

This first JNCC (JNCC I) was proposed in [9] and [10]. It is evaluated for two variants. JNCC Ia uses the UMTS turbo code and JNCC Ib uses a recursive-systematic (RCS) $\left[\begin{array}{ll}13 & 15\end{array}\right]_{8}$ convolutional code in the mobile stations. 


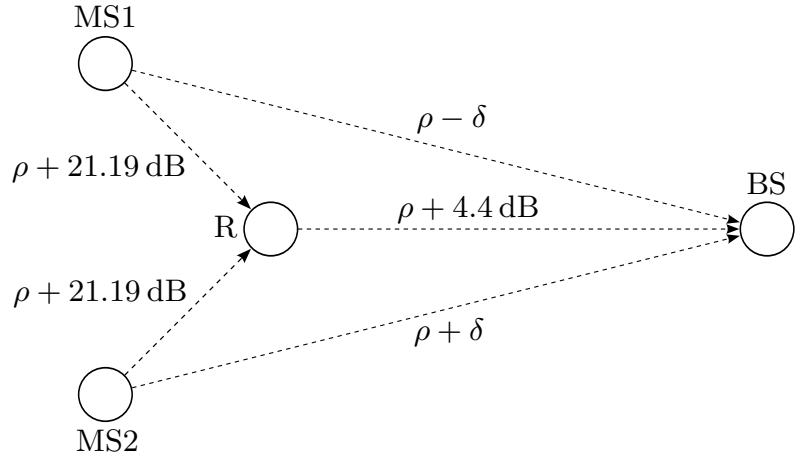

Figure 1: Multiple-access-relay-channel with two sources and one relay

The block diagram of the relay is shown in Figure 2(a). The estimates of the transmitted messages $\hat{\boldsymbol{u}}_{\mathrm{R}, 1}$ and $\hat{\boldsymbol{u}}_{\mathrm{R}, 2}$ are interleaved, where it is advantageous to choose a different interleaver than the interleaver in the turbo code, if a turbo code is used in the mobile stations [10]. As proposed in [10], we create a different interleaver by flipping the UMTS interleaver from left to right and call it $\Pi_{\mathrm{UTMS}}^{\mathrm{mod}}$. The interleaved bits are multiplexed into one bit sequence. When the length of both messages is equal, the bits appear alternately in the multiplexed sequence. For unequal message lengths a multiplexing scheme as follows can be used [10]: The interleaved bits are written row-by-row into a matrix with $\min \left(K_{1}, K_{2}\right)$ columns, where we write the bits of the smaller message at first. The last row of the matrix does not have to be completely filled. Now the bits are read column-by-column to obtain the multiplexed sequence. The multiplexed sequence is encoded using a recursive-systematic (RCS) $\left[\begin{array}{ll}13 & 15\end{array}\right]_{8}$ convolutional code and is punctured to length $M_{\mathrm{R}}$, where we transmit only parity bits, since the systematic bits are already included in the transmissions of the mobile stations. Then the sequence is modulated and transmitted.

The base station (see Figure 2(b)) receives three transmission, $\boldsymbol{y}_{\mathrm{D}, 1}$ and $\boldsymbol{y}_{\mathrm{D}, 2}$ from the mobile stations and $\boldsymbol{y}_{\mathrm{D}, \mathrm{R}}$ from the relay, which are demodulated to LLR's. The decoding is an iterative process which starts with the decoding of the transmissions received directly from the mobile stations. The decoder outputs are the LLR's of the information bits $\boldsymbol{L}_{\mathrm{MS} 1}$ and $\boldsymbol{L}_{\mathrm{MS} 2}$. When we subtract the a priori information ${ }^{1}$ that was fed into the SISO decoders, we obtain the extrinsic information $\boldsymbol{L}_{\mathrm{MS} 1}^{e}$ and $\boldsymbol{L}_{\mathrm{MS} 2}^{e}$. The extrinsic information vectors are interleaved and multiplexed in the same way as done in the relay. The resulting sequence is used as a priori information for the decoder of the relay transmission. The relay decoder improves the LLR's of information bits by using the relay transmission. The result is demultiplexed and the a priori information is subtracted to obtain the extrinsic information $\boldsymbol{L}_{\mathrm{R} 1}^{e}$ and $\boldsymbol{L}_{\mathrm{R} 2}^{e}$. These are used, after deinterleaving, as the a priori information for the decoders of the direct channel transmissions for the next iteration. After completing a sufficient number of iterations the hard estimates of the transmitted bits $\hat{\boldsymbol{u}}_{\mathrm{D}, 1}$ and $\hat{\boldsymbol{u}}_{\mathrm{D}, 2}$ are provided by the two decoders of the mobile station transmission. Note that the

\footnotetext{
${ }^{1}$ Note that for the first iteration the a priori information is zero.
}

estimates can also be obtained after the demultiplexing of the result of the relay decoder, considering that the results are still interleaved.

\subsection{JNCC II: XOR on the Code Bits}

The second scheme JNCC II is based on a code design presented in [11], where contrary to the setup in this work three mobile stations, that all want to exchange messages with the help of a relay, are considered. The block diagram of the relay and the base station for JNCC II are shown in Figure 3. We use the UMTS turbo code both in the mobile stations and in the relay and transmit all systematic bits and some of the parity bits chosen by a puncturing pattern.

In the relay the estimates $\hat{\boldsymbol{u}}_{\mathrm{R}, 1}$ and $\hat{\boldsymbol{u}}_{\mathrm{R}, 2}$ are reencoded by the same code as used in the corresponding mobile station ${ }^{2}$ and are punctured to obtain the two sequences $\boldsymbol{c}_{\mathrm{R} 1}$ and $\boldsymbol{c}_{\mathrm{R} 2}$ of the same length $M_{\mathrm{R}}$. Each of the sequences is interleaved by a different interleaver, where it is important to choose the interleavers in a way such that the serial combination of one interleaver and the other deinterleaver still forms a good interleaver. This demand emerges from the structure of the decoder, in which the soft information passes first one interleaver and is then deinterleaved by the other. We choose the interleavers as proposed in [11]: The first interleaver as the UMTS turbo code interleaver [17] $\Pi_{1}=\Pi_{\text {UMTS }}$ and the second as a serial combination of two times the first interleaver $\Pi_{2}=\Pi_{1} \Pi_{1}$. This way we have the serial combinations:

$$
\begin{aligned}
& \Pi_{1} \Pi_{2}^{-1}=\Pi_{\text {UMts }}\left(\Pi_{\mathrm{UMTS}} \Pi_{\mathrm{UMTS}}\right)^{-1}=\Pi_{\mathrm{UMTS}}^{-1} \\
& \Pi_{2} \Pi_{1}^{-1}=\left(\Pi_{\mathrm{UMTS}} \Pi_{\mathrm{UMTS}}\right) \Pi_{\mathrm{UMTS}}^{-1}=\Pi_{\mathrm{UMTS}}
\end{aligned}
$$

This means that the combinations lead to either the UMTS turbo code interleaver or its inverse and hence the soft information in the base station are interleaved or deinterleaved by $\Pi_{\text {Unts }}$, when passing from one decoder to the other. The interleaved sequences are bitwise XORed. This means that the network coding is not done on the information bits, but on the code bits. The resulting vector is then modulated and transmitted to the base station.

After demodulating and depuncturing the transmissions of the mobile stations (see Figure 3(b)) we have the LLR's of the code bits $\hat{\boldsymbol{c}}_{\mathrm{BS}, 1}^{d}$ and $\hat{\boldsymbol{c}}_{\mathrm{BS}, 2}^{d}$. We start by decoding the first of these sequences, although we could as well start with the second. The decoder are SISO decoders with the output of the a posteriori LLR's of the code bits. We puncture the extrinsic information of the decoder and interleave it afterwards using the puncturing scheme and interleaver as employed in the relay. To remove the contribution of the first mobile station from the relay transmission, we have to reverse the XOR operation of the relay, which would be again an XOR operation on binary values. For LLR's the analogy to the XOR operation is the box-plus operator [6]. We use the approximation also given in [6]:

$$
L_{1} \boxplus L_{2} \approx \operatorname{sgn}\left(L_{1}\right) \operatorname{sgn}\left(L_{2}\right) \min \left(\left|L_{1}\right|,\left|L_{2}\right|\right) .
$$

The LLR's of the relay transmission $\hat{\boldsymbol{c}}_{\mathrm{BS}, \mathrm{R}}$ and the contribution of MS1 are feed to the box-plus operator. The result is deinterleaved and depunctured to obtain the LLR $\hat{\boldsymbol{c}}_{\mathrm{BS}, 2}^{R}$, reversing the operations in the relay. Now $\hat{\boldsymbol{c}}_{\mathrm{BS}, 2}^{R}$ is added to

${ }^{2}$ Instead of reencoding the message a decoder that outputs a corrected encoded sequence can be used. 
(a)

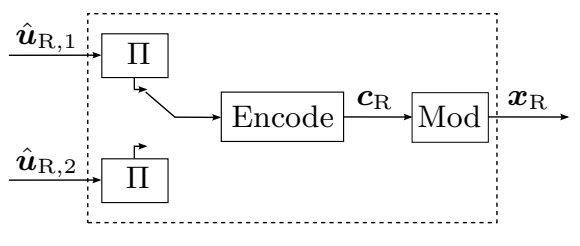

(b)

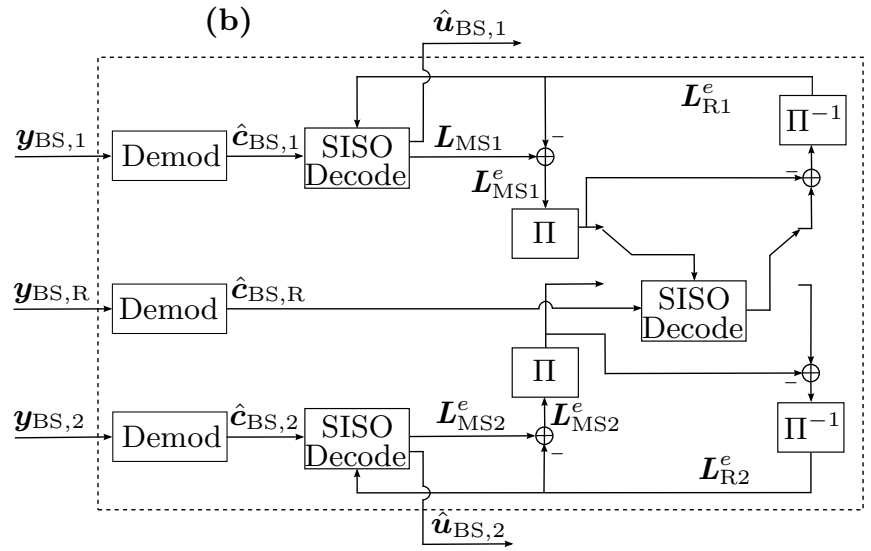

Figure 2: Block diagram of relay (a) and base station (b) for JNCC I

(b)

(a)

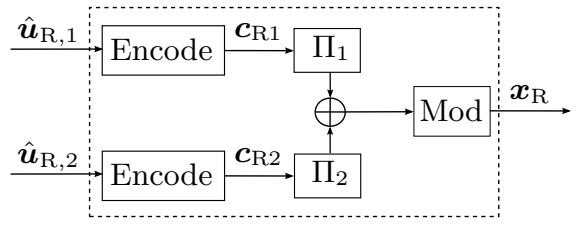

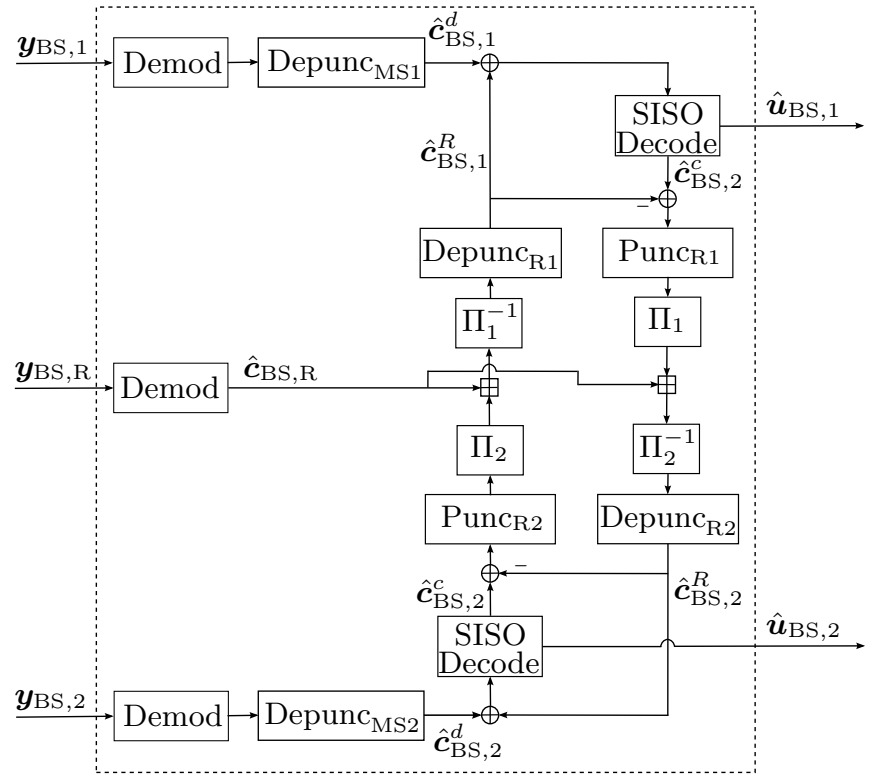

Figure 3: Block diagram of relay (a) and base station (b) for JNCC II

$\hat{\boldsymbol{c}}_{\mathrm{BS}, 2}^{d}$ and the sum is fed into the second decoder. The extrinsic information of the second decoder is used in the same way to retrieve the information about the first message $\hat{\boldsymbol{c}}_{\mathrm{BS}, 1}^{R}$ from the relay transmission. For the next iteration we add $\hat{\boldsymbol{c}}_{\mathrm{BS}, 1}^{R}$ to $\hat{\boldsymbol{c}}_{\mathrm{BS}, 1}^{d}$ and feed it to the first decoder. After a sufficient number of iterations we can obtain the hard estimates of the messages from the decoders.

\subsection{JNCC III: XOR on the Information Bits}

The next JNCC, referred to as JNCC III, is somewhat different to the other presented JNCC's. The network encoding is done on the information bits, keeping the layers of the OSI model apart, which means that we have no crosslayer encoding at the relay. The reason why we nevertheless call it a JNCC is that it employs a cross-layer decoding in the base station using the relay transmission as additional redundancy. Here again we use the UMTS turbo code in the mobile stations and the convolutional code in the relay.
In the relay (Figure 4(a)) the estimates $\hat{\boldsymbol{u}}_{\mathrm{R}, 1}$ and $\hat{\boldsymbol{u}}_{\mathrm{R}, 2}$ are interleaved by the interleavers ${ }^{3} \Pi_{1}=\Pi_{\mathrm{UTMS}}^{\mathrm{mod}}$ and $\Pi_{2}=\Pi_{1} \Pi_{1}$, where the construction of $\Pi_{\text {UTMS }}^{\mathrm{mod}}$ is given in 3.1. Without interleaving the performance is around $0.3 \mathrm{~dB}$ worse for the simulation parameters used in Section 5. After interleaving they are fed to a modulo 2-adder, an XOR block. If the messages have unequal lengths, the shorter message is zero padded before the XOR operation to the same length. The sum is encoded and punctured, where all systematic and some parity bits are transmitted.

A block diagram of the base station can be found in Figure 4(b). The iterative decoding is the same as JNCC I until the point when we have obtained the extrinsic information $\boldsymbol{L}_{\mathrm{MS} 1}^{e}$ and $\boldsymbol{L}_{\mathrm{MS} 2}^{e}$ from the direct transmissions. The extrinsic information is processed like the estimates of the messages in

\footnotetext{
${ }^{3}$ We can also use only one interleaver after the XOR block, if both interleavers before the XOR block $\Pi_{1}=\Pi_{2}$ are equal.
} 
(a)

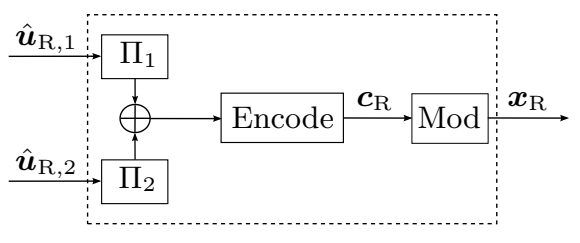

(b)

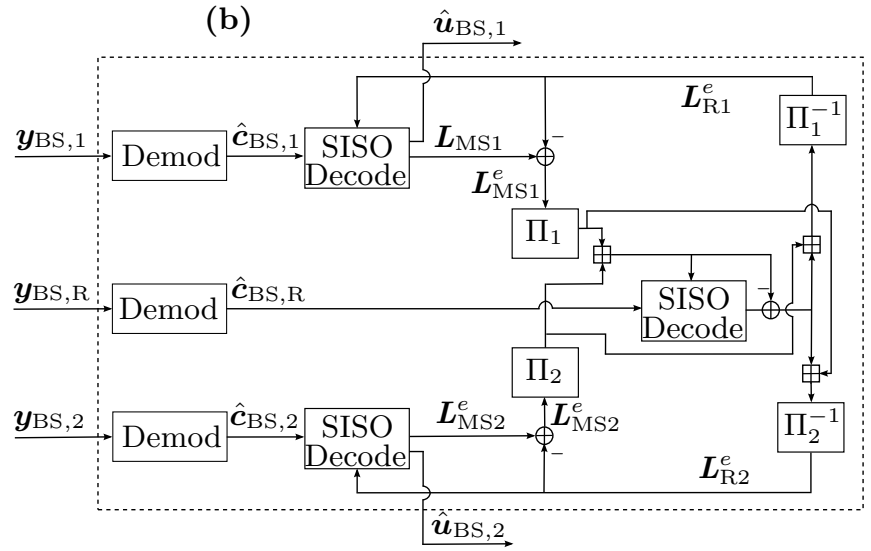

Figure 4: Block diagram of relay (a) and base station (b) for JNCC III

the relay: First each one is interleaved individually and then they are modulo 2-added, where the shorter message needs to be zero padded. The obtained sequence is used as a priori information for the SISO decoder of the relay transmission. To gain the extrinsic information of one sequence we need to feed the extrinsic information after the relay transmission decoder and the a priori information of the other sequence to a box-plus operator. The results of these operations are then deinterleaved, before which the padded zeros are removed. We obtain $\boldsymbol{L}_{\mathrm{R} 1}^{e}$ and $\boldsymbol{L}_{\mathrm{R} 2}^{e}$ which serve as a priori information for the next iteration. After iterating for a sufficient number of times, the results of the decoding process are taken as hard estimates $\hat{\boldsymbol{u}}_{\mathrm{BS}, 1}$ and $\hat{\boldsymbol{u}}_{\mathrm{BS}, 2}$ from the direct link decoders.

\subsection{JNCC IV: Duo Binary Encoder at Relay}

JNCC IV is similar to JNCC I. Again the UMTS turbo code is used at the mobile stations, but the code at the relay is different.

In the relay (see Figure $4(\mathrm{a})$ ) the estimates $\hat{\boldsymbol{u}}_{\mathrm{R}, 1}$ and $\hat{\boldsymbol{u}}_{\mathrm{R}, 2}$ are interleaved using equal interleavers $\Pi_{1}=\Pi_{2}=\Pi_{\mathrm{UTMS}}^{\mathrm{mod}}$. We construct one duo binary input from one bit of each of the estimates and fed it to a duo binary tail-biting encoder, which is used for example in WiMAX [2]. If the messages have different lengths, the shorter one is zero padded. From the duo binary encoder we obtain one encoded sequence which is punctured, modulated and sent to the base station. Note that we choose only parity bits for transmission in the relay.

The base station (Figure 4(b)) for JNCC IV is based on the decoder of JNCC I. We obtain the extrinsic information $\boldsymbol{L}_{\mathrm{MS} 1}^{e}$ and $\boldsymbol{L}_{\mathrm{MS} 2}^{e}$ in the same way as for JNCC I. The extrinsic information are interleaved using the same interleavers as in the relay. The interleaved LLR's, plus some zero padding if necessary, are then used as a priori information for the duo binary SISO decoder of the relay transmission. The output of the decoder are two information bit LLR vectors (one for each message). To use only the extrinsic information we need to subtract from each its corresponding a priori information. After deinterleaving the vectors are used as the a priori information $\boldsymbol{L}_{\mathrm{R} 1}^{e}$ and $\boldsymbol{L}_{\mathrm{R} 2}^{e}$ for the next iteration. After sufficient iterations the hard decision results $\hat{\boldsymbol{u}}_{\mathrm{BS}, 1}$ and $\hat{\boldsymbol{u}}_{\mathrm{BS}, 2}$ are taken from the mobile station decoders, where these results could also be taken in an interleaved form the relay decoder.

\subsection{Comparison of Support for Asymmetric Rates}

Although we only investigate equally sized messages from MS1 and MS2, a comparison of the support for asymmetric rates is interesting. The support is realized in different ways: JNCC I uses a multiplexing scheme. JNCC III and JNCC IV use zero padding to increase the length of the shorter message. Those two ways of support asymmetric rates imply that both messages are protected by the same degree in the relay transmission. JNCC II uses an approach which protects the shorter message by a higher degree than the longer one. It uses puncturing, after which the code rate of the shorter message is lower. The different degrees of protection could be helpful with asymmetric rate setups.

\section{REFERENCE SYSTEMS}

We compare the JNCC schemes to the following reference systems. The first two reference system do not use networkcoding and the last one does use both network and channelcoding, but not jointly.

\subsection{Point-To-Point Channels}

This reference system (P2P) does not use the relay at all, but uses only the two direct point-to-point channels. Hence we only have two time slots. The total number of symbols is $M_{\text {total }}=M_{\mathrm{P} 2 \mathrm{P}, 1}+M_{\mathrm{P} 2 \mathrm{P}, 2}$. We use the UMTS turbo code in both mobile stations.

\subsection{Relay Channels}

The next reference system (RC) consists of two detached relay channels. The relay slot is split, where each part is used to support the transmission of one of the mobile stations ${ }^{4}$. Hence this system would also be applicable for two spatially distributed relays. Since the relay processes each message individually, but does not combine them, this is a form of routing. We use the UMTS turbo code both in mobile station and relay and use complementary puncturing

${ }^{4}$ The total number of symbols of the relay is given as $M_{\mathrm{R}}=$ $M_{\mathrm{R}, 1}+M_{\mathrm{R}, 2}$. 
(a)

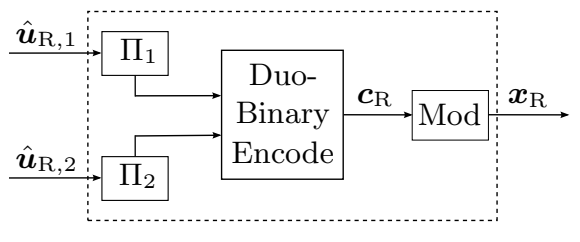

(b)

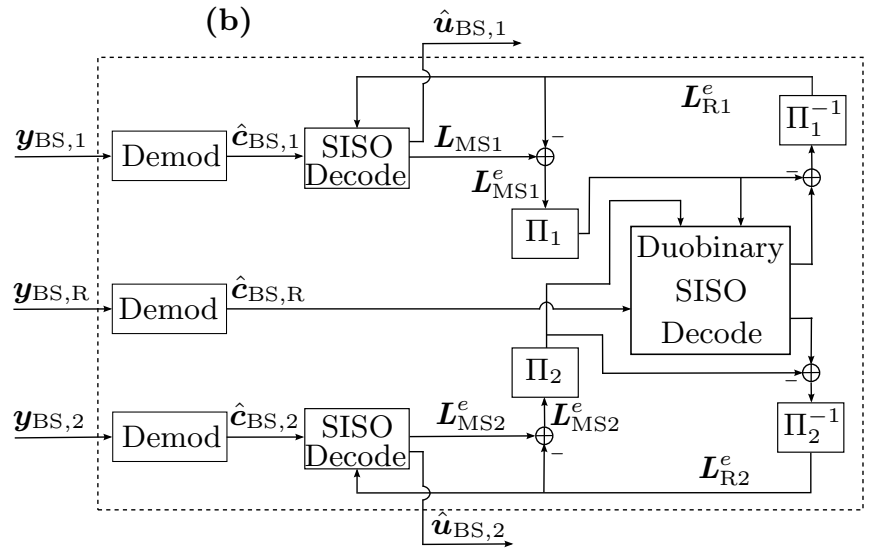

Figure 5: Block diagram of relay (a) and base station (b) for JNCC IV

patterns like suggested in [16]. It is also possible to use convolutional codes and to establish a distributed turbo code, like proposed in [18] and [23].

\subsection{Separate-Network-Channel-Code}

The separate-network-channel-code (SNCC), presented for example in [3], is a code that makes use of network-coding and channel-coding, but does not use both techniques jointly. The physical and the network layer are not mixed and can be treated separately. This is an advantage over JNCC, where the two layers are merged into one and have to be considered jointly.

In the mobile station and the relay we use the UMTS turbo code. Using the CRC after the decoding we can create erasure-based links between the nodes on the network layer.

The relay of SNCC is similar to JNCC III. The difference is that we do not use interleavers. If correctly decoded, the estimates of the messages are XORed $\boldsymbol{u}_{\mathrm{R}}=\boldsymbol{u}_{1} \oplus \boldsymbol{u}_{2}$, using zero padding when the messages have unequal lengths. After channel encoding all systematic and some parity bits are transmitted.

In the base station the three transmission from the mobile stations are separately decoded, where each decoder makes a hard decision on the output. Now the integrity of the decoding results can be checked from the CRC. If both messages where decoded successfully no further action is necessary. If one of the two messages was decoded incorrectly, it can be retrieved from the relay transmission, given that the relay transmission was decoded successfully. The erroneous messages is obtained from the modulo 2-sum of the other message and the relay message, where zero padding is used if necessary. For example if $\hat{\boldsymbol{u}}_{\mathrm{BS}, 1}$ is decoded incorrectly, it can be retrieved as $\hat{\boldsymbol{u}}_{\mathrm{BS}, 1}=\hat{\boldsymbol{u}}_{\mathrm{BS}, 2} \oplus \hat{\boldsymbol{u}}_{\mathrm{BS}, \mathrm{R}}$.

\section{SIMULATION RESULTS WITHOUT AN OPTIMIZED ALLOCATION}

In this section we present the simulation results for equally distributed symbol allocations, meaning that the same number of symbols is allocated to a mobile station and to a relay. The total number of symbols per packet transmission is fixed for all systems to $M_{\text {total }}=6000$ to enable a fair comparison. Hence we have: $M_{1}=M_{2}=M_{\mathrm{R}}=2000$ for JNCC, $\mathrm{SNCC}$ and $\mathrm{RC}^{5}$ and $M_{\mathrm{P} 2 \mathrm{P}, 1}=M_{\mathrm{P} 2 \mathrm{P}, 2}=3000$ for $\mathrm{P} 2 \mathrm{P}$. We use a symmetric rate with packets of $K_{1}=K_{2}=1500$ information bits. Then the total rate of the system is given as $R_{\mathrm{S}}=1 / 2$. The number of iterations of the iterative decoding in the base station is set to 4 . We call these iterations outer iterations as opposed to the inner iterations of a turbo decoder. For the demodulation perfect channel state information (CSI) is assumed and the output LLR's are not quantized. The average SNR between a mobile station and the relay is $\rho+21.19 \mathrm{~dB}$ and between relay and base station $\rho+4.4 \mathrm{~dB}$.

We show theoretical outage rates and common packet error rates (CPER). The theoretical outage rates, which are a theoretical bound, are obtained as explained in [10]. The conditions for an outage of JNCC are given in Appendix A. A common packet error occurs when at least one of the two messages cannot be retrieved correctly. We plot the theoretical bounds as dashed lines and the CPER as solid lines, if not stated otherwise.

First we look in Figure 6 onto the results for the symmetric setup, i.e. $\delta=0 \mathrm{~dB}$. The upper bounds predict the trend of the practical systems. JNCC performs best with a gap of $3 \mathrm{~dB}$ to SNCC, which both provide a diversity order of 2 , as can be observed from the slope of the curves. Of the practical systems JNCC I shows the best results. Of the two variants, JNCC Ib works better than JNCC Ia. The second best scheme is JNCC II. JNCC IV works worse than JNCC II for lower SNR's, but for higher SNR's it seems to be slightly better than JNCC II, but still worse than JNCC I. The idea behind IV and I is similar and the advantages and disadvantages are the same, but JNCC I shows better results. JNCC III performs worst, but still outperforms SNCC, due to the decoding with iterative exchange of soft information. Numerical values of the gaps for the practical JNCC's from the theoretical bound are given in Table 5. Whereas in the practical systems that use network coding a diversity gain is obtain, in the other reference systems, $\mathrm{RC}$ and $\mathrm{P} 2 \mathrm{P}$, no diversity gain is achieved for the given parameters.

${ }^{5}$ For $\mathrm{RC}$ the relay transmission is $\operatorname{split}\left(M_{\mathrm{R}, 1}=M_{\mathrm{R}, 2}=\right.$ 1000). 


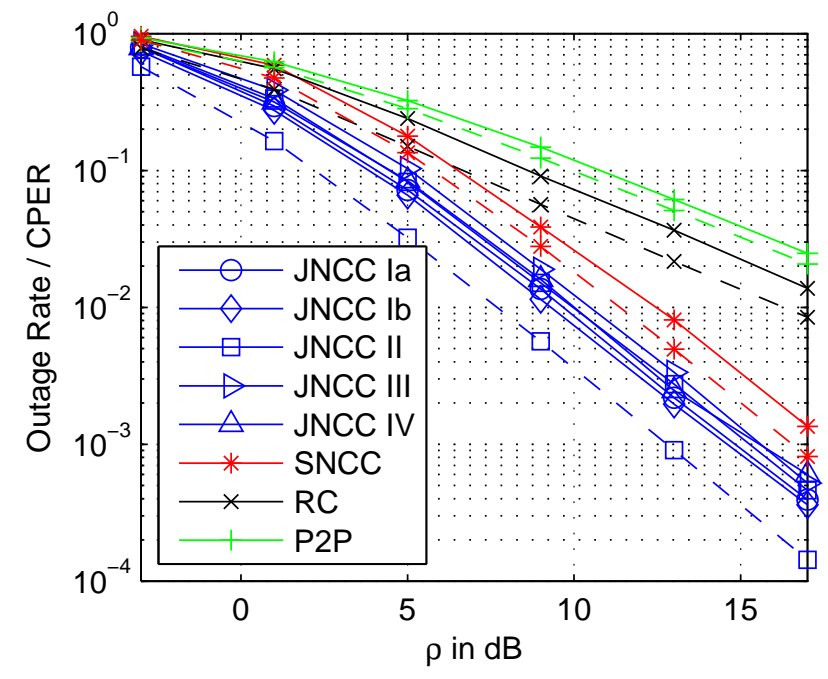

Figure 6: Theoretical bounds and simulation results for the symmetric setup (Solid lines: Practical systems; Dashed lines: Theoretical bounds)

\begin{tabular}{|l|r|}
\hline System & Gap at $\rho=9 \mathrm{~dB}$ \\
\hline JNCC Ia & $2 \mathrm{~dB}$ \\
JNCC Ib & $1.5 \mathrm{~dB}$ \\
JNCC II & $2.25 \mathrm{~dB}$ \\
JNCC III & $2.75 \mathrm{~dB}$ \\
JNCC IV & $2.35 \mathrm{~dB}$ \\
\hline
\end{tabular}

Table 1: Gap to the theoretical bound for the practical JNCC's systems for the symmetric setup

Next we examine in Figure 7 the theoretical upper bounds for asymmetric setups. For higher SNR values the slope of the curves for the asymmetric cases are the same as for the symmetric case, but the curves are shifted to the right. With increasing asymmetry the gap to the symmetric setup increases for all considered systems. The increase of the outage rates is greater for JNCC than for SNCC. The reason why SNCC loses less is that for greater asymmetries we have more often the case where one transmission can be decoded without error and the other has to be retrieved from the relay transmission, for which SNCC is designated. This means that the additional redundancy for both messages, that JNCC provides, is less often needed. For the reference systems, which do not use network-coding, the performance loss is greater, since no diversity is achieved for the chosen simulation parameters. The performance of the practical systems, follows the trend of the theoretical upper bounds also for asymmetric setups. The practical JNCC's perform within $3 \mathrm{~dB}$ for medium and higher SNR's for asymmetries up to $\delta=12 \mathrm{~dB}$. Interesting is that the gap is similar for the considered asymmetries and that the order of the different practical JNCC schemes remains the same.

\section{OPTIMIZATION OF SYMBOL ALLO- CATION}

After having examined the results for equally allocated symbols, the question arises whether the results can be improved

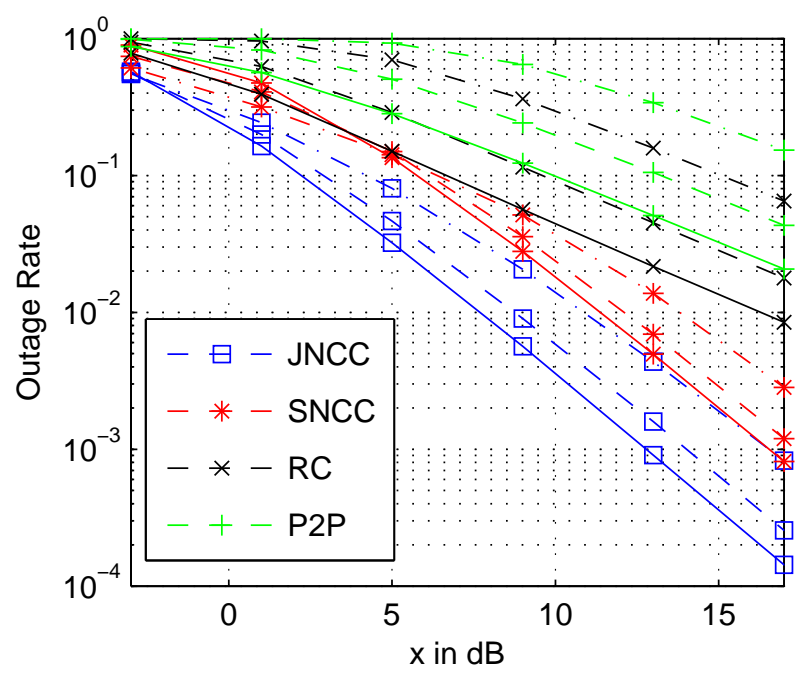

Figure 7: Theoretical bounds for different asymmetries(Solid lines: $\delta=0 \mathrm{~dB}$; Dashed lines: $\delta=6 \mathrm{~dB}$; Dashed-dotted lines: $\delta=12 \mathrm{~dB}$ )

by an optimized symbol allocation. We try to minimize the outage rates of JNCC for Rayleigh channels given certain fixed rates. We show two approaches: One which needs instantaneous channel state information (CSI) at the transmitter and an approach which bases on only statistical knowledge of the channel coefficients.

\subsection{Optimization with Instantaneous Channel State Information}

An ideal symbol allocation method would consider the actual channel coefficients at each time instant. This requires instantaneous CSI not only at the relay and the base station, but also at the mobile stations. The approach is basically an optimization for AWGN channels with the instantaneous SNR's as average SNR's of the AWGN channels. A reliable communication is either possible via the direct channels or via the relay.

The minimum symbol allocation to enable a reliable communication via the direct channels can be obtained easily. From inequalities for the direct channels in Appendix A, which are tight for the minimum, we can conclude

$$
\begin{array}{r}
M_{1}^{\dagger}=\frac{K_{1}}{C\left(\gamma_{1 \mathrm{D}}\right)} \quad M_{2}^{\dagger}=\frac{K_{2}}{C\left(\gamma_{2 \mathrm{D}}\right)} \\
\text { s.t. } \quad M_{1}^{\dagger}+M_{1}^{\dagger} \leq M_{\text {total }}, \quad M_{1}^{\dagger} \in \mathbb{N}, \quad M_{2}^{\dagger} \in \mathbb{N},
\end{array}
$$

as the minimum number of symbols to transmit the messages reliably over the direct channels.

We use linear programming to find a minimum symbol allocation which fulfills the inequalities of the theoretical outage rates for the transmission via the relay. The optimization is

$$
\boldsymbol{x}^{*}=\arg \min _{\boldsymbol{x}} \boldsymbol{f}^{T} \boldsymbol{x},
$$


where $\boldsymbol{x}^{*}, \boldsymbol{x}$ and $\boldsymbol{f}$ are defined as

$$
\boldsymbol{x}^{*}=\left[\begin{array}{c}
M_{1}^{*} \\
M_{2}^{*} \\
M_{\mathrm{R}}^{*}
\end{array}\right], \quad \boldsymbol{x}=\left[\begin{array}{c}
M_{1} \\
M_{2} \\
M_{\mathrm{R}}
\end{array}\right], \quad \boldsymbol{f}=\left[\begin{array}{l}
1 \\
1 \\
1
\end{array}\right] .
$$

The cost function could be defined differently if we do not assume the same costs for transmitting a symbol from a mobile station or from the relay. The optimization is subject to the constraints given by

$$
\boldsymbol{A} \boldsymbol{x} \geq \boldsymbol{b}, \quad \boldsymbol{x} \in \mathbb{N}^{3},
$$

where $\boldsymbol{A}$ and $\boldsymbol{b}$ are defined as

$$
\boldsymbol{A}=\left[\begin{array}{ccc}
C\left(\gamma_{1 \mathrm{R}}\right) & 0 & 0 \\
0 & C\left(\gamma_{2 \mathrm{R}}\right) & 0 \\
C\left(\gamma_{1 \mathrm{D}}\right) & 0 & C\left(\gamma_{\mathrm{RD}}\right) \\
0 & C\left(\gamma_{2 \mathrm{D}}\right) & C\left(\gamma_{\mathrm{RD}}\right) \\
C\left(\gamma_{1 \mathrm{D}}\right) & C\left(\gamma_{2 \mathrm{D}}\right) & C\left(\gamma_{\mathrm{RD}}\right) \\
-1 & -1 & -1
\end{array}\right]
$$

and

$$
\boldsymbol{b}=\left[\begin{array}{c}
K_{1} \\
K_{2} \\
K_{1} \\
K_{2} \\
K_{1}+K_{2} \\
-M_{\text {total }}
\end{array}\right]
$$

Note that only integer allocations are allowed, as fractions of a symbol cannot be allocated. When both optimizations are infeasible, no reliable communication with JNCC can be realized for the given parameters and channel coefficients and an outage occurs. Usually the required number of symbols is smaller than $M_{\text {total }}$, which means that the rate could be increased for the particular channel coefficients. It is also possible to find closed form expressions of the maximum rate for a given rate ratio and average channel SNR's. This leads to different cases, depending on which of the inequalities are tight, where in [9] the expressions for some of the cases are given.

\subsection{Optimization with Statistical Channel Knowledge}

It is more realistic to assume that no CSI is available at the transmitter, but the statistical distribution of the channel coefficients and the average SNR and hence the distribution of instantaneous SNR is known. An optimized symbol allocation can be found with the help of a Monte-Carlo optimization. We use of five random generators with the characteristics of the Rayleigh channels to create coefficient sets. For these coefficient sets we can calculate channel capacities sets for an average SNR value. Now we determine for each possible symbol allocation ${ }^{6}$ the number of outage events of

${ }^{6}$ The quantity of symbol allocations $\left[M_{1}, M_{2}, M_{\mathrm{R}}\right]$ is bounded by the following inequalities:

$$
\begin{aligned}
\frac{K_{1}}{L_{1}} & \leq M_{1} \leq M_{\text {total }}-\frac{K_{2}}{L_{2}} \\
\frac{K_{2}}{L_{2}} & \leq M_{2} \leq M_{\text {total }}-\frac{K_{1}}{L_{1}} \\
0 & \leq M_{\mathrm{R}} \leq M_{\text {total }}-M_{1}-M_{2} .
\end{aligned}
$$

the theoretical bound from the equations in Appendix A. The symbol allocation which causes the least outage events is the optimal allocation for the generated channel constellations sets.

This optimization yields $M_{1}=M_{2}=1723$ and $M_{\mathrm{R}}=2554$ for the symmetric case and $M_{1}=2083, M_{2}=1601$ and $M_{\mathrm{R}}=2316$ for $\delta=6 \mathrm{~dB}$ respectively, both at $\rho=9 \mathrm{~dB}$. We see that the most symbols are allocated to the relay transmission. This can be explained by the look onto the parameters of the system model as given in Section 5. The average SNR of the channels from the mobile stations to the relay is much higher than the average MS-BS SNR $\rho$. Hence only fewer additional parity bits are needed to ensure a reliable transmission to the relay. For setups with different parameters the results of the optimization would probably produce other optimized allocations.

\section{SIMULATION RESULTS WITH OPTI- MIZED ALLOCATIONS}

In this section we use the same simulation parameter as in Section 5, but use the optimized allocations. We show the information theoretic outage rates in Figure 8 for the symmetric setup. The channel allocation optimized with instantaneous CSI shows the best performance. Note that for higher average SNR's the gap to the not optimized allocation increases. This is due to more freedom when optimizing the symbol allocation. For higher SNR values we need less error correction redundancy as the channels are less noisy and hence have more freedom in choosing a symbol allocation to combat the channel fading. The performance gain using the allocation optimized with statistical channel knowledge is smaller and around $0.3 \mathrm{~dB}$ for all SNR values. For asymmetric setups the results are similar.

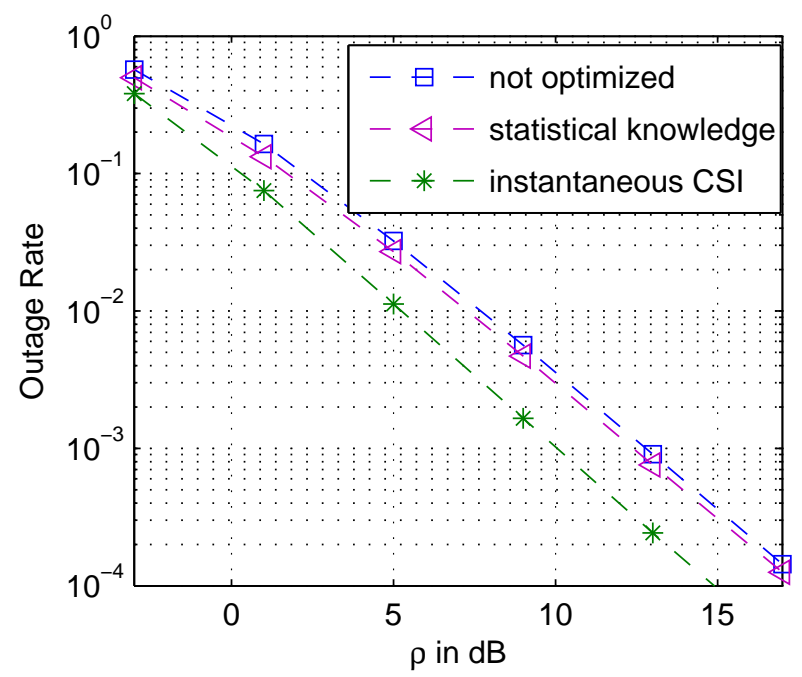

Figure 8: Theoretical bounds for JNCC for $\delta=0 \mathrm{~dB}$ with allocations, that are not optimized $\left(M_{1}=M_{2}=\right.$ $\left.M_{\mathbf{R}}=2000\right)$, optimized using statistical knowledge $\left(M_{1}=2083, M_{2}=1601, M_{\mathbf{R}}=2316\right)$ and optimized for instantaneous CSI.

The gain in performance of the practical JNCC's from us- 
ing the not optimized allocation to using the symbol allocations optimized with statistical channel knowledge is shown in Figure 9 for an asymmetry of $\delta=6 \mathrm{~dB}$. A positive gain of at least $0.3 \mathrm{~dB}$ for JNCC Ia/b and JNCC II is obtained. For JNCC III the gain is negative. Where of course a problem is that the optimization is not done for a practical JNCC scheme, but for the theoretical bounds. For a symmetric setup the gain is negative or very low for all JNCC's.

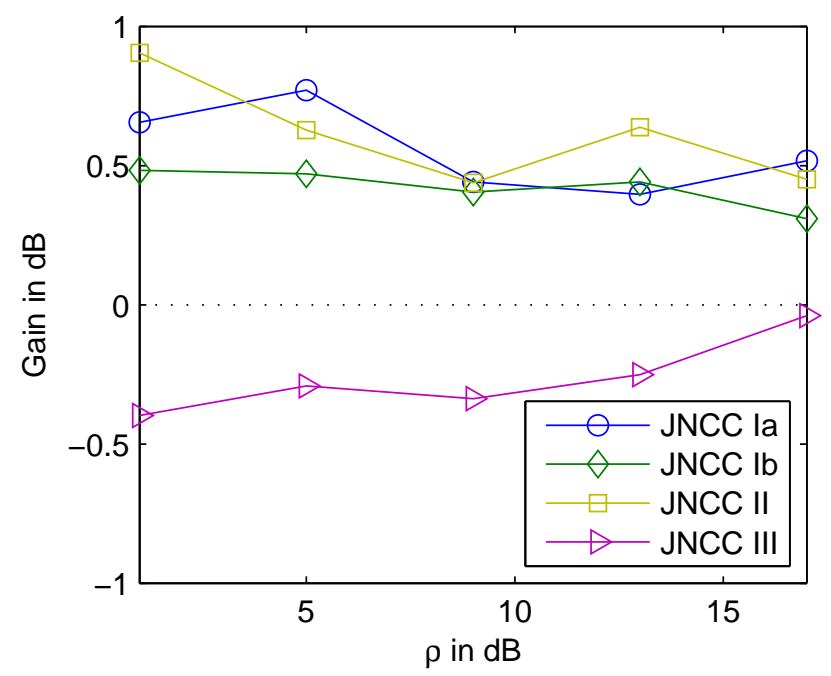

Figure 9: Gain of JNCC's for an allocation optimized with statistical channel knowledge $\left(M_{1}=2083\right.$, $\left.M_{2}=1601, M_{\mathbf{R}}=2316\right)$ from the not optimized allocation $\left(M_{1}=M_{2}=M_{\mathbf{R}}=2000\right)$ for asymmetric channels with $\delta=6 \mathrm{~dB}$

\section{CONCLUSIONS}

We examined four JNCC's, where two are novel approaches, and three references systems for the MARC with block Rayleigh fading channels. We have showed that JNCC performs better than the reference systems and can increase the diversity order. Of the reference systems only SNCC can gain diversity for the chosen system parameters. JNCC I performs best. JNCC III performs worst of the JNCC's, but still outperforms SNCC and offers the advantage of no crosslayer encoding in the relay. With increasing asymmetry the performance becomes worse, but the order of the different systems remains the same. The performance loss for asymmetric channel setups is much greater for systems without diversity gain. All JNCC schemes perform within $3 \mathrm{~dB}$ of the theoretical upper bound for all considered asymmetries up to $\delta=12 \mathrm{~dB}$. The optimization of the symbol allocation with statistical channel knowledge at the transmitter leads to a gain of the JNCC theoretical upper bounds compared to the not optimized allocation. For an asymmetric channel setup a performance gain for JNCC I and JNCC III can be achieved. But before considering using an optimized allocation, further research is recommended, since the gain is negative for a symmetric MARC.

In this work we investigated only symmetric rates. In future work the performance of the presented schemes for asymmetric rates could be analyzed. The JNCC's could be modified to improve the support of asymmetric rates. Especially for asymmetric rates optimizations of the allocation, where the rates are not fixed, are interesting. Another interesting aspect, are setups with more than two mobile stations and/or more than one relay.

\section{ACKNOWLEDGMENTS}

This works is supported by the Space Agency of the German Aerospace Center and the Federal Ministry of Economics and Technology with the support code 50 YB 0905 and by the German Ministry of Education and Research in the framework of the Alexander von Humboldt-Professorship.

\section{REFERENCES}

[1] R. Ahlswede, N. Cai, S.-Y. R. Li, and R. W. Yeung. Network information flow. IEEE Transactions on Information Theory, 46(4):1204-1216, July 2000.

[2] J. G. Andrews and A. G. and R. Muhamed. Fundamentals of WiMAX. Pearson Education, Inc., New Jersey, 2007.

[3] Y. Chen, S. Kishore, and J. Li. Wireless diversity through network coding. In IEEE Wireless Communications and Networking Conference (WCNC), pages 1681-1686. IEEE, April 2006.

[4] T. M. Cover and J. A. Thomas. Elements of information theory. John Wiley \& Sons, Inc., New Jersey, 2006.

[5] D. Duyck, D. Capirone, M. Moeneclaey, and J. Boutros. A full-diversity joint network-channel code construction for cooperative communications (pimrc). In IEEE 20th International Symposium on Personal Indoor and Mobile Radio Communications, pages 1282-1286. IEEE, September 2009.

[6] J. Hagenauer, E. Offer, and L. Papke. Iterative decoding of binary block and convolutional codes. IEEE Transactions on Information Theory, 42(2):429-445, March 1996.

[7] A. Hatefi, R. Visoz, and A. Berthet. Joint channel-network coding for the semi-orthogonal multiple access relay channel. In IEEE 72nd Vehicular Technology Conference Fall (VTC 2010-Fall), pages 1-5. IEEE, September 2010.

[8] A. Hatefi, R. Visoz, and A. Berthet. Joint channel-network turbo coding for the non-orthogonal multiple access relay channel. In IEEE 21st International Symposium on Personal Indoor and Mobile Radio Communications (PIMRC), pages 408-413. IEEE, September 2010.

[9] C. Hausl. Joint Network-Channel Coding for Wireless Relay Networks. Dissertation, Technische Universität München.

[10] C. Hausl. Joint network-channel coding for the multiple-access relay channel based on turbo codes. European Transactions on Telecommunications, 20:175-181, January 2009.

[11] O. Iscan, I. Latif, and C. Hausl. Network coded multi-way relaying with iterative decoding. In IEEE 21st International Symposium on Personal Indoor and Mobile Radio Communications (PIMRC), pages 482-487. IEEE, September 2010.

[12] G. Kramer, M. Gastpar, and P. Gupta. Cooperative strategies and capacity theorems for relay networks. 
IEEE Transactions on Information Theory, 51(9):3037-3063, September 2005.

[13] G. Kramer and A. J. van Wijngaarden. On the white Gaussian multiple-access relay channel. In IEEE International Symposium on Information Theory, page 40. IEEE, June 2000.

[14] M. D. Renzo, L. Iwaza, M. Kieffer, P. Duhamel, and K. A. Agha. Robust wireless network coding - a survey. In The 2nd International ICST Conference on Mobile Lightweight Wireless Systems. ISCT, May 2010.

[15] L. Sankaranarayanan, G. Kramer, and N. B. Mandayam. Hierarchical sensor networks: capacity bounds and cooperative strategies using the multiple-access relay channel model. In First Annual IEEE Communications Society Conference on Sensor and Ad Hoc Communications and Networks (SECON), pages 191-199. IEEE, October 2004.

[16] M. Souryal and B. Vojcic. Cooperative turbo coding with time-varying Rayleigh fading channels. In IEEE International Conference on Communications (ICC), pages 356-360. IEEE, June 2004.

[17] M. Valenti and J. Sun. The UMTS turbo code and an efficient decoder implementation suitable for software-defined radios. International Journal of Wireless Information Networks, 8(4):203-215, October 2001.

[18] M. Valenti and B. Zhao. Distributed turbo codes: towards the capacity of the relay channel. In IEEE 58th Vehicular Technology Conference (VTC), pages 322-326. IEEE, October 2003.

[19] T. Wong. Numerical calculation of symmetric capacity of Rayleigh fading channel with BPSK/QPSK. IEEE Communications Letters, 5(8):328-330, August 2001.

[20] L. Xiao, D. Costello, and T. Fuja. Network coded cooperative diversity with multiple sources. In IEEE Global Telecommunications Conference (GLOBECOM), pages 1-5. IEEE, December 2009.

[21] L. Xiao, T. Fuja, J. Kliewer, and D. Costello. A network coding approach to cooperative diversity. IEEE Transactions on Information Theory, 53(10):3714-3722, October 2007.

[22] S. Yang and P. Koetter. Network coding over a noisy relay : a belief propagation approach. In IEEE International Symposium on Information Theory (ISIT), pages 801-804. IEEE, June 2007.

[23] B. Zhao and M. Valenti. Distributed turbo coded diversity for relay channel. Electronics Letters, 39(10):786-787, May 2003.

\section{APPENDIX \\ A. THEORETICAL BOUNDS OF JNCC}

The theoretic bounds were derived in [10] and can be obtained by the use of cut-set-bounds [4]. An outage event OUT is defined as the case where a reliable communication from at least one of the sources to its destination is impossible. The outage rates given here are applicable for time-division multiple access, decode-and-forward and a relay that remains silent on decoding errors, like used throughout this work.

The capacity for an instantaneous SNR $\gamma$ is returned by the function $C(\gamma)$. For a Gaussian distributed channel input it is given as $C(\gamma)=\log _{2}(1+\gamma)$. For discrete modulation alphabets the function is not available as an closed form expression and has to be evaluated numerically. Methods can be found for example in [19].

The event no outage for JNCC is given by

$$
\overline{\mathrm{OUT}}_{\mathrm{JNCC}}=\overline{\mathrm{OUT}}_{\mathrm{JNCC}}, \vee \overline{\mathrm{OUT}}_{\mathrm{P} 2 \mathrm{P}} \text {. }
$$

$\overline{\mathrm{OUT}}_{\mathrm{JNCC}}$, is the event that a communication using the relay is possible and is calculated as

$$
\begin{aligned}
& \overline{\mathrm{OUT}}_{\mathrm{JNCC}}= \\
& \quad\left[K_{1} \leq M_{1} C\left(\gamma_{1 \mathrm{R}}\right)\right] \wedge\left[K_{2} \leq M_{2} C\left(\gamma_{2 \mathrm{R}}\right)\right] \\
& \wedge {\left[K_{1} \leq M_{1} C\left(\gamma_{1 \mathrm{D}}\right)+M_{\mathrm{R}} C\left(\gamma_{\mathrm{RD}}\right)\right] } \\
& \wedge {\left[K_{2} \leq M_{2} C\left(\gamma_{2 \mathrm{D}}\right)+M_{\mathrm{R}} C\left(\gamma_{\mathrm{RD}}\right)\right] } \\
& \wedge {\left[K_{1}+K_{2} \leq M_{1} C\left(\gamma_{1 \mathrm{D}}\right)+M_{2} C\left(\gamma_{2 \mathrm{D}}\right)+M_{\mathrm{R}} C\left(\gamma_{\mathrm{RD}}\right)\right] . }
\end{aligned}
$$

The first line is the event that the relay can decode both messages correctly and transmit a combination of both signals to the base station. The other lines determine whether the base station can decode both messages based on the two transmissions from the mobile stations and the transmission from the relay. When the transmission via the relay fails, a communication only over the direct channels is still possible if the following inequality is fulfilled

$$
\overline{\mathrm{OUT}}_{\mathrm{P} 2 \mathrm{P}},=\left[K_{1} \leq M_{1} C\left(\gamma_{1 \mathrm{D}}\right)\right] \wedge\left[K_{2} \leq M_{2} C\left(\gamma_{2 \mathrm{D}}\right)\right] .
$$

In [10] also the conditions for an outage event of each reference system are given. The author also shows, that with JNCC or SNCC diversity can be gained for higher rates and for a greater variety of rate and allocation pairs. 